\title{
Losartan was more effective than atenolol for isolated systolic hypertension and left ventricular hypertrophy
}

Kjeldsen SE, Dahlöf B, Devereux RB, et al. Effects of losartan on cardiovascular morbidity and mortality in patients with isolated systolic hypertension and left ventricular hypertrophy: a Losartan Intervention for Endpoint Reduction (LIFE) substudy.JAMA 2002;288:1491-8.

\section{QUESTION: In patients with isolated systolic hypertension (ISH) and left ventricular hypertrophy (LVH), is losartan based therapy more effective than atenolol based therapy?}

\section{Design}

Randomised \{allocation concealed*\}, blinded \{patients, clinicians, data collectors, outcome assessors, data analysts, and manuscript writers $\} \nmid{ }^{*}$ controlled trial with mean 4.7 year follow up.

\section{Setting}

945 outpatient settings in Europe and the US.

\section{Patients}

1326 patients between 55 and 80 years of age (mean age $70 \mathrm{y}, 60 \%$ women) with ISH (sitting blood pressure [BP] 160-200 mm Hg systolic and $<90 \mathrm{~mm} \mathrm{Hg}$ diastolic after 1-2 wk of placebo) and electrocardiographic signs of LVH. Follow up was $99.8 \%$

\section{Intervention}

Patients were allocated to once daily losartan based therapy $(n=660)$ or atenolol based therapy $(n=666)$ with hydrochlorothiazide as the second agent in both groups to reach a target systolic BP $<140 \mathrm{~mm} \mathrm{Hg}$.

\section{Main outcome measures}

A composite endpoint of cardiovascular mortality, myocardial infarction (MI), and stroke. Secondary outcomes included all cause mortality and new onset diabetes mellitus.

\section{Main results}

Analysis was by intention to treat. After adjustment for degree of LVH and Framingham risk score (sex, cholesterol, high density lipoprotein cholesterol, smoking, presence of diabetes and LVH, systolic BP, and body mass index) at baseline, groups did not differ for the composite endpoint (table) or MI. Cardiovascular mortality, stroke, all cause mortality, and new onset diabetes occurred less frequently in patients who received losartan than in those who received atenolol (table).

\section{Conclusion}

In patients with isolated systolic hypertension and left ventricular hypertrophy, losartan reduced cardiovascular mortality, stroke, all cause mortality, and new onset

*See glossary.

†Information provided by author.

\section{COMMENTARY}

In the study by Kjeldsen $e t$ al of patients with ISH and electrocardiographic evidence of LVH, blockade of the renin angiotensin system with the angiotensin receptor blocker losartan reduced cardiovascular events more than did the $\beta$ blocker atenolol, despite similar BP lowering. Given that the goal of antihypertensive therapy is to prevent cardiovascular events, this shows that the choice of antihypertensive agent is just as important as lowering BP in ISH.

Although losartan reduced the rate of stroke and cardiovascular mortality more than did atenolol, noticeably absent in these results was any treatment benefit on MI. Kjeldsen $e t$ al propose that "cardioprotective" actions of $\beta$ blockers may also extend to hypertension. However, the Medical Research combination of hydrochlorothiazide and amiloride reduced MI by $24 \%$, but the $\beta$ blocker atenolol showed no substantial reduction despite similar levels of $\mathrm{BP}$ lowering and the fact that $43 \%$ of the participants in the MRC trial had ISH. Because the MRC trial suggests that atenolol was not as "cardioprotective" as hydrochlorothiazide combined with amiloride, further study is probably required. In the meantime, we no longer have to treat hypertension with $\beta$ blockers as if it was "pre" coronary disease.

Christopher M Rembold, MD University of Virginia Charlottesville, Virginia, USA

1 Medical Research Council trial of treatment of hypertension in older adults: principal results. MRC Working Party. BMJ 1992;304:405-12. diabetes more than atenolol. Council (MRC) trial in older adults ${ }^{1}$ found that the diuretic
Source of funding:

Merck E Co.

For correspondence

Dr S E Kjeldsen,

Ullevaal Hospital, Oslo

Norway.

sverre.kjeldsen@

ioks.uiono

Abstract and commentary also appear in ACP Journal Club

IICardiovascular mortality, stroke, and myocardial infarction. 